\title{
Abiraterone acetate and docetaxel with androgen deprivation therapy in high-volume metastatic hormone-sensitive prostate cancer in China: an indirect treatment comparison and cost analysis
}

\author{
Xin $\mathrm{Hu}^{1}$, Shuli $\mathrm{Qu}^{2}$, Xingxing Yao ${ }^{3}$, Chaoyun $\mathrm{Li}^{3}$, Yanjun $\mathrm{Li}^{2}$ and Jianye Wang ${ }^{4^{*}}$
}

\begin{abstract}
Background: To conduct an indirect treatment comparison of patients with high-volume mHSPC and a cost analysis between Abi-ADT and Doc-ADT therapies in China.

Methods: The Bucher technique for indirect treatment comparison was used. A cost analysis was conducted from both healthcare and patient perspectives.

Results: The indirect treatment comparison demonstrated no significant difference in PFS for Abi-ADT versus Doc-ADT (HR: 0.84, 95\% Cl 0.66-1.07). Doc-ADT therapy costs less than Abi-ADT, with potential savings of up to RMB 887,057 per patient from the healthcare perspective and RMB 226,210 per patient from the patient perspective.

Conclusions: No significant differences in PFS between Doc-ADT and Abi-ADT therapy for patients with high-volume $\mathrm{mHSPC}$. Doc-ADT therapy is a cost-saving alternative to Abi-ADT in China.
\end{abstract}

Keywords: Prostate cancer, Docetaxel, Abiraterone, Cost analysis

\section{Background}

Prostate cancer is the most common cancer for men worldwide [1]. It also ranks fifth and tenth in estimated deaths worldwide and in China, respectively [2]. The incidence rates of prostate cancer within the Asian population have historically been lower than those in the Western population; however, in recent years, the incidence and mortality rate in China have grown rapidly, with a three-fold increase in the last decade. This dramatic rise in incidence has led to 25,000 deaths annually with an estimated 5-year survival rate of 54\% [3, 4].

In China, due to the low rate of prostate-specific antigen screening to detect cancer at an early stage, a panel of

\footnotetext{
*Correspondence: wangjy@bjhmoh.cn

${ }^{4}$ Department of Urology, Beijing Hospital, No. 1 Dongdan Dahua Road, Dongcheng District, Beijing 100730, China

Full list of author information is available at the end of the article
}

local clinical experts reported that most Chinese patients at the time of diagnosis had high-volume metastatic hormone-sensitive prostate cancer (mHSPC), which included the presence of visceral metastases, a bonemetastasis burden categorised by site (beyond the axial skeleton) or by a high number of lesions, or a combination of these [5].

The treatment for men with mHSPC has historically included androgen deprivation treatment (ADT), which can be accomplished by surgical castration or medical suppression of testicular function with synthetic analogues of gonadotropin-releasing hormone [6]. The addition of docetaxel or abiraterone acetate plus prednisone to ADT has been recommended for patients with newly diagnosed mHSPC [7]. The CHAARTED [5], LATITUDE [8] and GETUG-AFU-15 [8,9] trials demonstrated that the addition of docetaxel and abiraterone acetate to ADT improved overall survival (OS) and progression-free 
survival (PFS) among men with mHSPC compared with ADT alone. However, no head-to-head clinical trials have compared abiraterone acetate plus prednisone/prednisolone with ADT (Abi-ADT) to docetaxel with ADT (Doc-ADT). A recently published network meta-analysis (NMA) conducted by Wallis in 2017 revealed no significant difference in OS for men treated with Abi-ADT versus Doc-ADT. However, the pooled result of PFS was not reported. A growing belief among the oncology community is that delaying the progression of metastatic disease is a worthwhile goal, even without improvement in OS [10]. Based on the above rationale, the Wallis study must be updated with the PFS outcomes associated with Doc-ADT and Abi-ADT for patients with high-volume mHSPC. With rapidly increasing healthcare demands and limited medical resources in China, medical institutions are confronted with the urgent task of controlling medical expenses. Chinese national level medical spending exceeded significantly that of all G7 members except the US in terms of current purchase power parity [11]. In addition, there is an ongoing public debate about the effective introduction and spreading of value based medicine concepts, and introduction of cost-effectiveness criteria into official policy making in most world regions [12]. Therefore, the application of Doc-ADT and Abi-ADT therapies should be carefully considered by balancing clinical outcomes and related costs. No economic data comparing these two therapies in China are available.

In summary, the main objective of our study is to determine the comparative efficacy and costs of Doc-ADT and Abi-ADT therapies in the treatment of patients with high-volume mHSPC. To achieve this goal, we first updated the NMA outcomes of the two treatments. We then performed an economic evaluation comparing DocADT and Abi-ADT therapies in China through a cost analysis from both healthcare and patient perspectives.

\section{Indirect treatment comparison}

\section{Identification of eligible trials and data extraction}

Studies of adults with high-volume mHSPC comparing the efficacy of Doc-ADT or Abi-ADT to ADT alone were identified. Only randomised controlled trials (RCTs) were included. PubMed and Cochrane Library were searched for trials published in English, and the Chinese databases CNKI and WanFang were searched for studies published in Chinese. Search terms were derived from the Wallis study and updated from August to November 2017. Table 1 summarises the PICOS (population, intervention, controls, outcomes, and setting) elements corresponding to the research questions. The primary endpoints were OS and PFS.

Two reviewers (SQ and YL) independently extracted all data from the eligible trials. For each included trial, we extracted the characteristics of the participants and the interventions, efficacy and safety outcomes, the sample size (randomised and analysed) in each arm, numerical results, and loss to follow-up data.

We assessed the risk of bias in each included trial using the Cochrane Collaboration's Risk of Bias tool [13].

\section{Statistical methods}

The Kaplan-Meier (KM) survival data of Doc-ADT and Abi-ADT were reconstructed by applying Guyot's method [14], which uses digital software to read in the coordinates of the KM curves from the published graph and uses the information on numbers at risks, often published at four or five time points under the $x$-axis of the KM graph, and total number of events, to reconstruct the Kaplan-Meier data. We used this method to reconstruct the KM survival data for Doc-ADT and Abi-ADT arms from the corresponding $\mathrm{KM}$ survival curves in CHAARTED [15] and Fizazi's [8] trials, respectively.

The hazard ratio (HR) of response to Doc-ADT and Abi-ADT versus ADT alone was calculated

Table 1 PICOS statement for the inclusion and exclusion criteria

\begin{tabular}{lll}
\hline & Inclusion & Exclusion \\
\hline $\begin{array}{ll}\text { Study population } \\
\text { Intervention }\end{array}$ & $\begin{array}{l}\text { Patients with high-volume metastatic hormone-sensitive pros- } \\
\text { tate cancer, regardless of age, sex, ethnic group or disease } \\
\text { status }\end{array}$ & Any not listed in the inclusion criteria \\
Comparator & $\begin{array}{l}\text { Docetaxel + ADT } \\
\text { Abiraterone + prednisone + ADT }\end{array}$ & Any not listed in the inclusion criteria \\
Autcome measures & Clinical efficacy outcomes Safety outcomes & Any not listed in the inclusion criteria \\
Study design & Randomised clinical trials (RCTs) & Any not listed in the inclusion criteria \\
Restrictions & Full-text published manuscripts in English or Chinese & Editorials OR Notes OR Comments OR Letters OR Case reports OR \\
& Year limitation: up to Nov 2017 & Pharmacokinetic studies OR Epidemiology studies \\
& & Not full-text published manuscripts Non-English or non-Chinese \\
& & studies
\end{tabular}


independently using Review Manager 5.3 (The Nordic Cochrane Centre, The Cochrane Collaboration, Copenhagen, Denmark) software. A random effect model was used due to the clinical heterogeneity inherent in the data. Heterogeneity among the studies was assessed using Chi square and $I^{2}$ statistics. The pooled results are presented in the form of forest plots.

An indirect treatment comparison was performed to assess the clinical effects of Doc-ADT and Abi-ADT using ADT as the common comparator arm. We used Bucher's successive pairwise approach [16], which has been identified as an appropriate method for performing indirect treatment comparisons of HRs [17]. The indirect treatment comparison was conducted using Excel 2013 (Microsoft Inc, Richmond, WA, USA).

\section{Indirect treatment comparison results Evidence synthesis}

Among 235 identified studies, 4 papers from 3 clinical trials were eligible for inclusion: the CHAARTED [5, 15], LATITUDE [8], and GETUG-AFU15 trials [9]. Patients in the LATITUDE trial generally had high-volume $\mathrm{mHSPC}$ as $96 \%$ of the patients had bone lesions with Gleason scores $\geq 8$ and $\geq 3$. Compared with the Wallis study, we also extracted the PFS data of patients with high-volume mHSPC from these 4 papers.

Overall, 1895 patients with high-volume mHSPC were included in the analysis; 354 (18.7\%) patients received Doc-ADT, 597 (31.5\%) received Abi-ADT, and 944 (49.8\%) received ADT alone.

\section{Characteristics and quality of the included studies}

In the Doc-ADT trials (CHAARTED and GETUG-AFU $15)$, the patients in the experimental arm received docetaxel $75 \mathrm{mg} / \mathrm{m}^{2}$ for a maximum of six [15] or nine [9] cycles. In the Abi-ADT trial (LATITUDE), the patients in the experimental arm received abiraterone acetate $1000 \mathrm{mg} / \mathrm{d}$ with prednisone $5 \mathrm{mg} / \mathrm{d}$ [8]. The quality of all included studies was assessed, and a low risk of bias with adequate randomisation was indicated (Fig. 1).

\section{OS}

The Wallis study conducted subgroup analyses of OS results of patients with high-volume mHSPC. In our study, we used the same methods for subgroup analyses and obtained equivalent but more detailed results. The pooled HRs assessing OS among patients with highvolume mHSPC were 0.68 (95\% confidence interval [CI] $0.52-0.87, I^{2}=24 \%$, heterogeneity Chi square $\mathrm{p}=0.25,2$ trials, 696 patients) for Doc-ADT versus ADT alone and 0.60 (95\% CI 0.48-0.75, 1 trial, 1199 patients) for Abi$\mathrm{ADT}$ versus ADT alone (Fig. 2a). The indirect treatment comparison of Abi-ADT versus Doc-ADT indicated no significant difference in OS (HR: 0.88, 95\% CI 0.62-1.25; Fig. 2a).

\section{PFS}

The pooled HRs assessing PFS among patients with highvolume mHSPC were 0.56 (95\% CI $0.46-0.67, I^{2}=0 \%$, heterogeneity Chi square $\mathrm{p}=0.48,2$ trials, 696 patients) for Doc-ADT versus ADT alone and 0.47 (95\% CI 0.400.55, 1 trial, 1199 patients) for Abi-ADT versus ADT alone (Fig. 2b). The indirect comparison of Abi-ADT versus Doc-ADT indicated no significant difference in PFS (HR: 0.84, 95\% CI 0.66-1.07; Fig. 2b).

\section{Cost analysis}

The above results combined with those of the Wallis study demonstrated no significant differences in OS and PFS between patients with high-volume mHSPC treated with Doc-ADT and those treated with Abi-ADT. Based on the equivalent efficacy between Doc-ADT and AbiADT, we conducted a cost analysis to compare the local drug and medical costs between these two therapies in China.

\section{Model overview}

A decision-analytic model was built to simulate the disease process of mHSPC and estimate the comparative costs of Doc-ADT and Abi-ADT treatment regimens in a Chinese setting.

Patients with mHSPC were included in the model. Based on disease progression, we defined three health states in the model: progression-free survival (PFS), progressed disease (PD) and death. The model began with 1000 patients. At any given time, a patient would be in one of the three states. A patient could remain in PFS (or PD) or advance to PD (or death) during each cycle, and the patient sample was followed until death. The length of each cycle was 1 month. Direct medical costs were considered, including the costs of the drugs (docetaxel, abiraterone, and prednisolone), hospitalisation costs for chemotherapy, ADT cost per month, laboratory test costs, and costs associated with adverse events (AEs). All costs were discounted 3\% annually. Model development and the data analysis were conducted using Microsoft ${ }^{\circledR}$ Excel 2013 (Microsoft, Redmond, WA, USA).

\section{Model input}

The numbers of surviving patients, patients with clinical progression and deceased patients each month were obtained by fitting OS and PFS survival curves of the CHAARTED trials ADT arm. Parametric functions were assessed based on model goodness of fit using Akaike Information Criterion (AIC) and Bayesian Information Criterion (BIC) as well as a visual assessment of each 


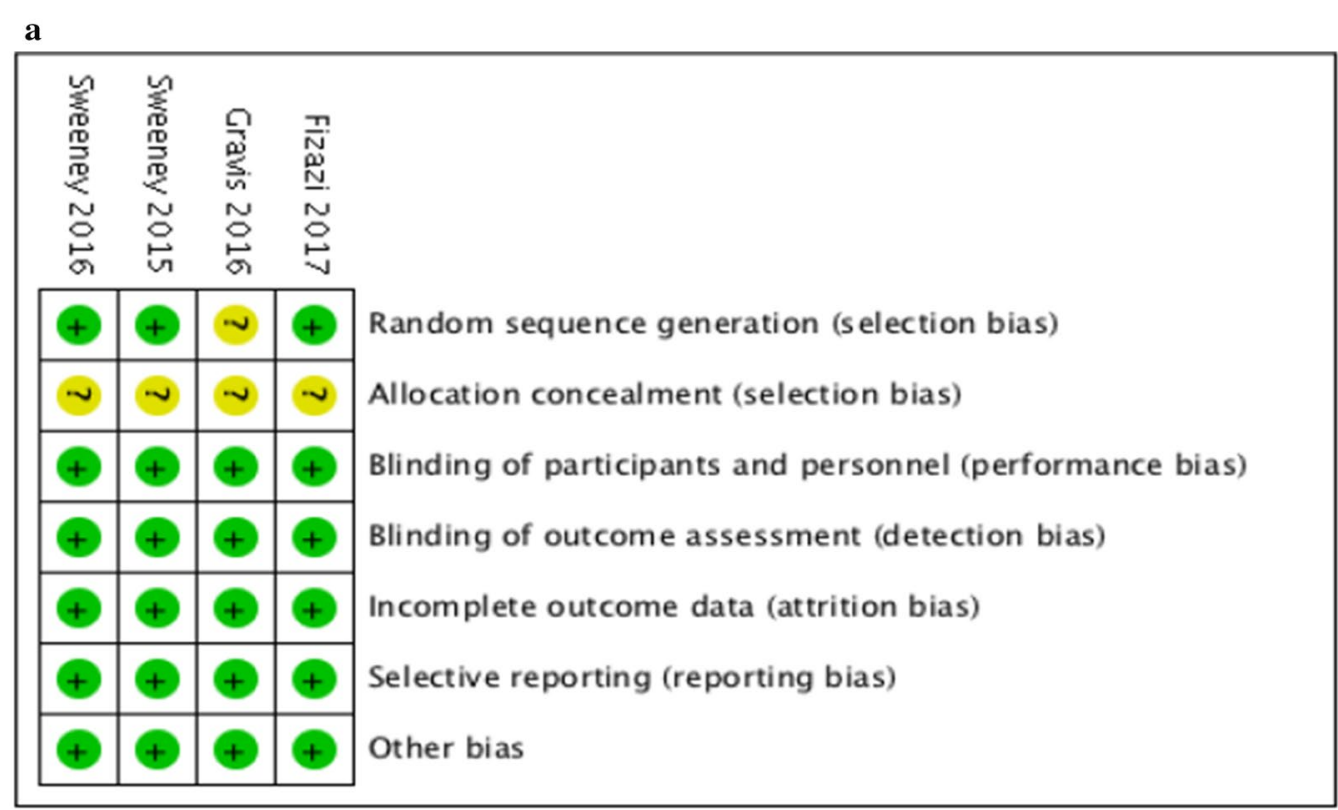

b

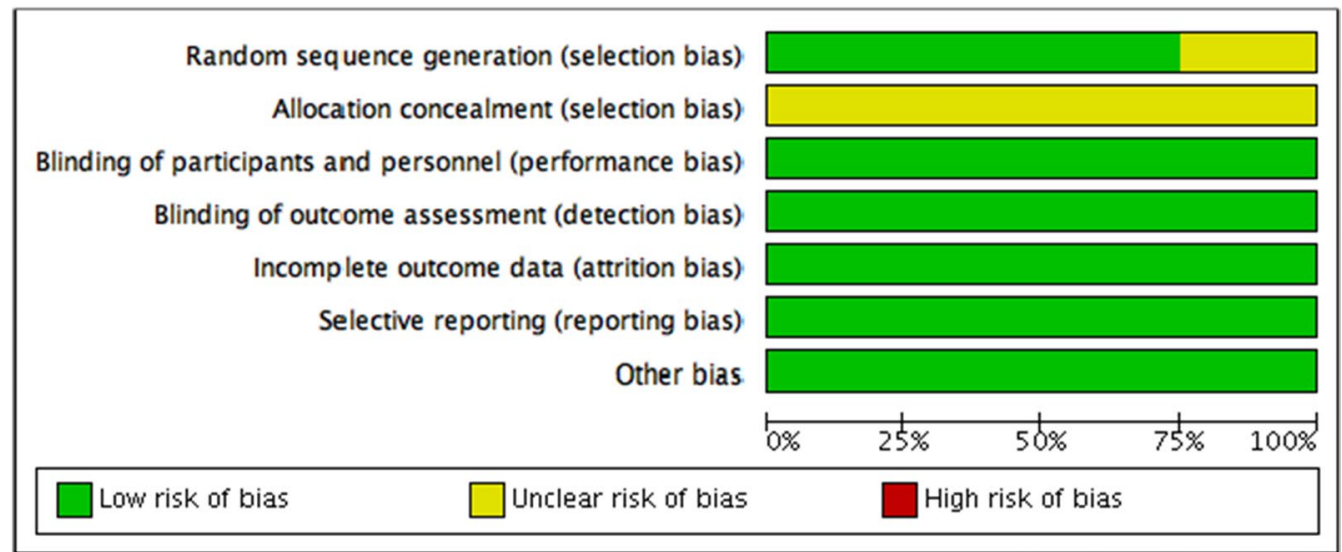

Fig. 1 Risk of bias assessment of the included studies: a risk of bias graph and $\mathbf{b}$ risk of bias summary

parametric function. Log-logistic and Log-normal distribution were selected for the OS and PFS curve respectively based lowest AIC and BIC value. Then, the HRs from NMA were applied to reconstruct the curve for Abi-ADT and Doc-ADT arms (Fig. 3).

For patients with clinical progression, the second-line treatment varied widely across the two therapies. Based on the Sweeney 2015 study [5], 54 of 180 (34\%) patients with progression receiving Doc-ADT therapy continued docetaxel treatment, while the remaining patients $(66 \%)$ were switched to abiraterone. According to Chinese experts' opinions, the clinical protocols for patients with progression in China are similar. Among the patients receiving Abi-ADT therapy, all patients with progression were switched to docetaxel treatment for 10 cycles.
The unit costs of docetaxel and prednisolone/prednisone were extracted from the IQVIA China Hospital Pharmaceutical Audit (CHPA) database, which was updated in the first three quarters of 2017. Because abiraterone acetate has been included in the national negotiation drug list in China, we used the negotiated price as its drug cost. Weighted average dosages from the included trials were calculated and entered into the cost analysis. Body surface area (BSA, $\mathrm{cm}^{2}$ ) was calculated as $0.0061 \times$ height $(\mathrm{cm})+0.0128 \times$ weight $(\mathrm{kg})-0.1529$. An average height of $161.5 \mathrm{~cm}$ and an average weight of $61.8 \mathrm{~kg}$, reflecting a typical Chinese adult [18], were suggested by local clinical experts (Table 2).

In addition to the costs of docetaxel, prednisolone/ prednisone and abiraterone acetate associated with 


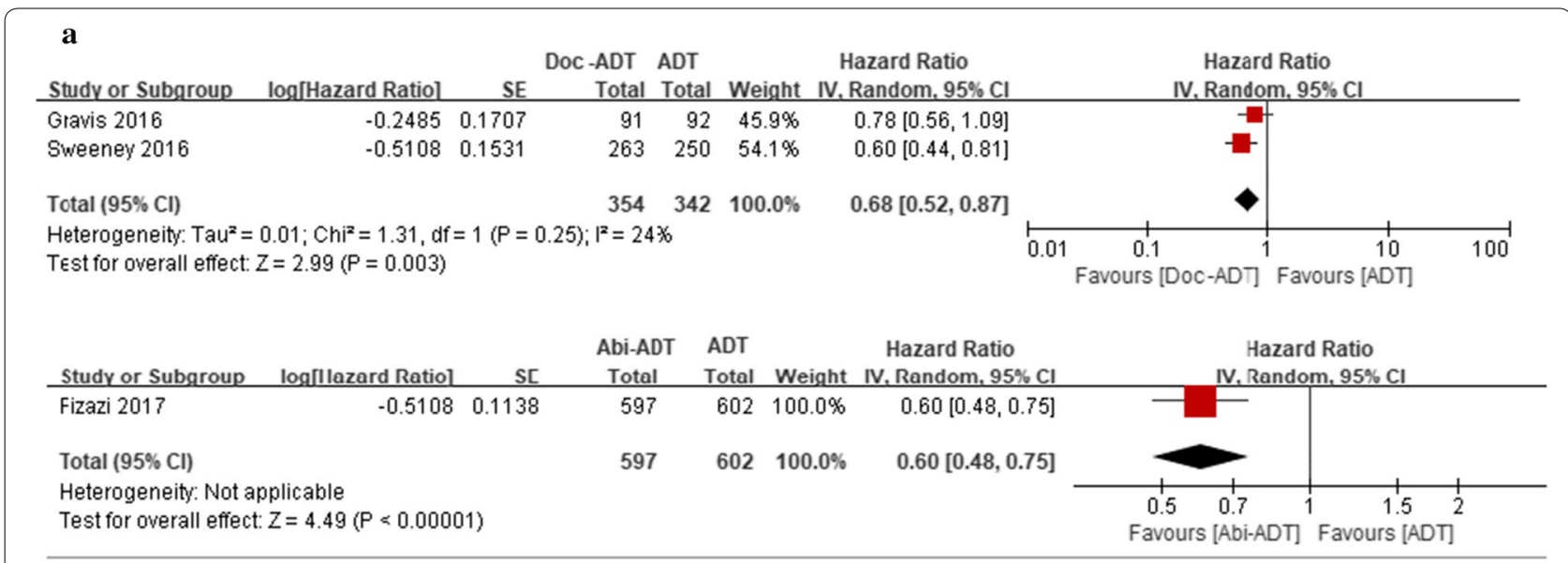

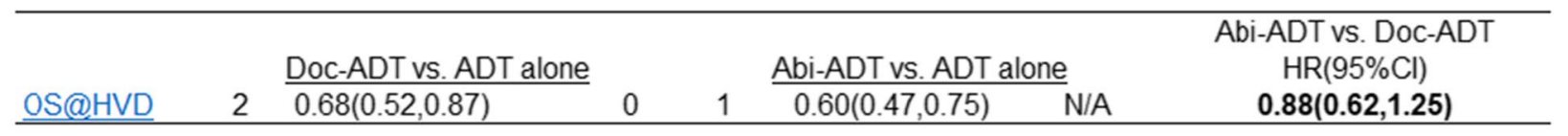

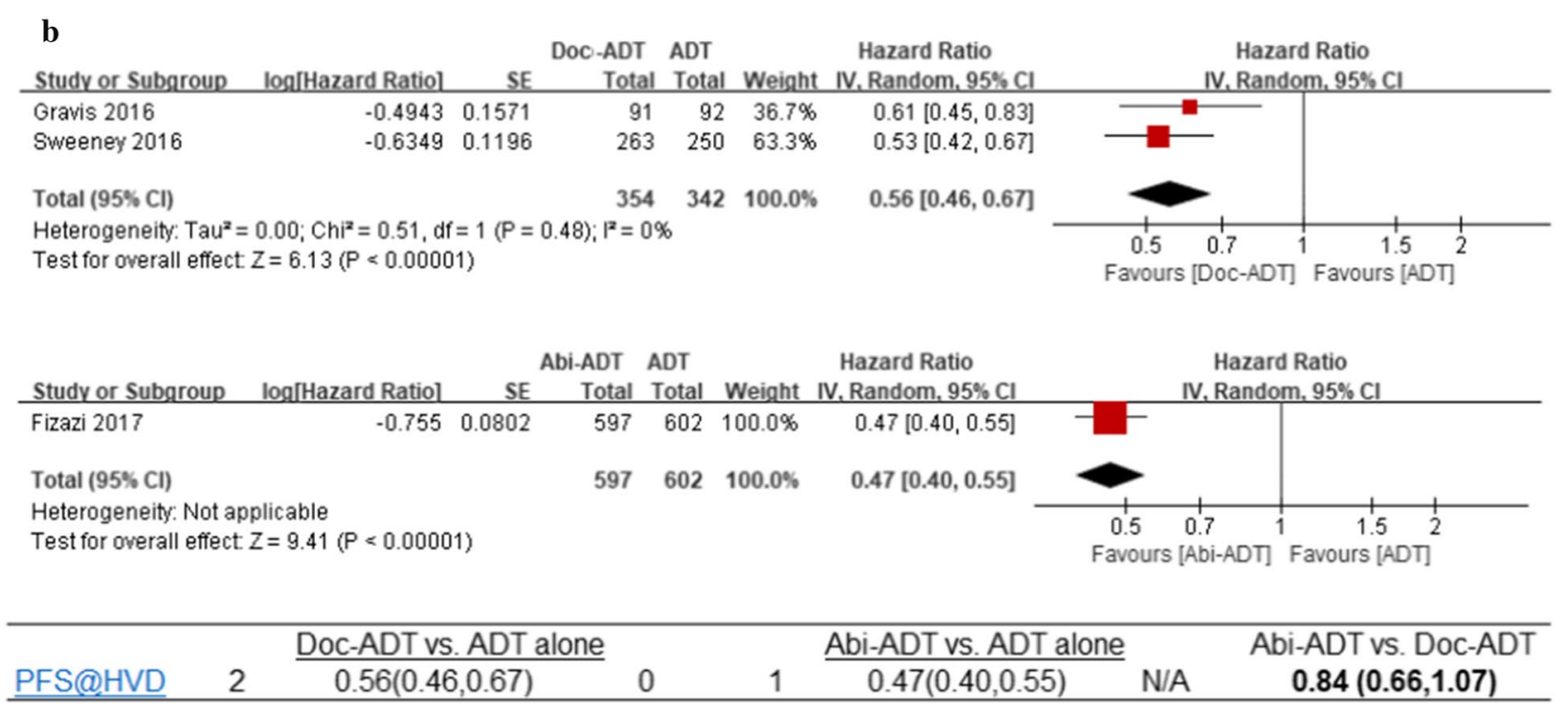

Fig. 2 Forest plot for the meta-analysis of combination therapy versus ADT alone with respect to: $\mathbf{a}$ overall survival (OS) and $\mathbf{b}$ progression-free survival (PFS). Abi abiraterone acetate, Cl confidence interval, $d f$ degree of freedom, Doc docetaxel, HR hazard ratio, $I V$ instrumental variables, SE standard error

Doc-ADT and Abi-ADT therapies, interviews with clinicians from five major cities in China (Beijing, Shanghai, Wuhan, Chengdu and Guangzhou) were conducted to collect information regarding other medical resource use and cost data related to the two therapies. Other costs included the hospitalisation costs for chemotherapy, ADT cost per month, laboratory test costs, and the costs associated with AEs. Patients receiving docetaxel required hospitalisation, while patients treated with abiraterone acetate did not. The hospitalisation costs for patients receiving docetaxel included hospital bed fees and the cost of preventive drugs for chemotherapy. All patients required regular examinations, including routine blood tests, blood biochemistry and liver function tests. The incidence of AEs was obtained from the included clinical trials and is listed in the Table 3. 


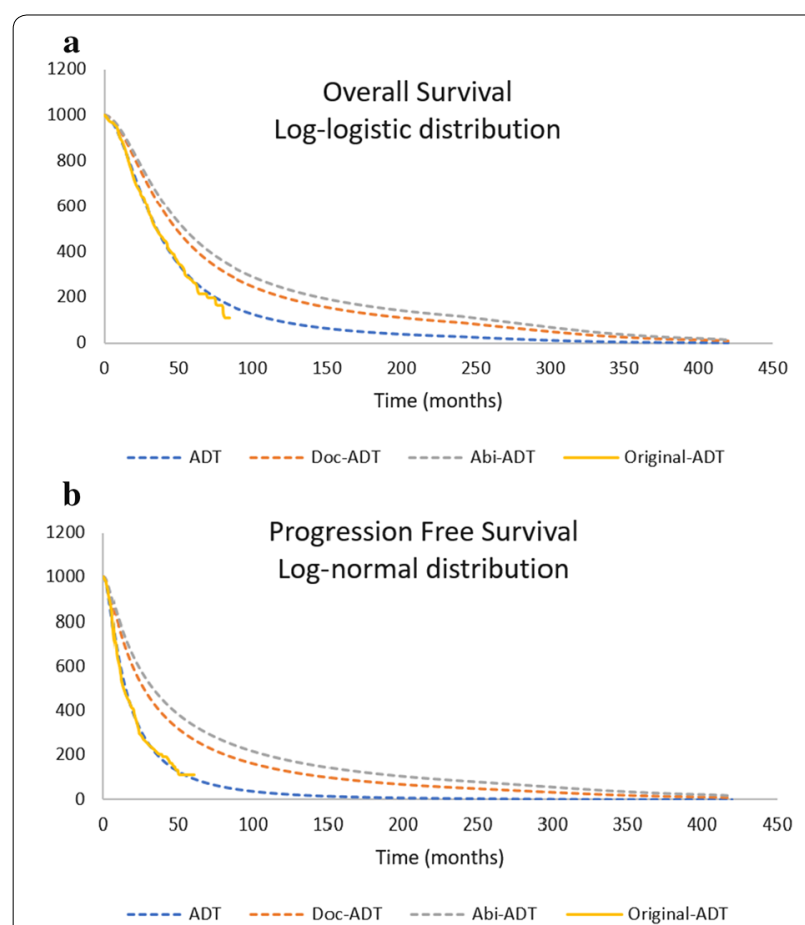

Fig. 3 Parametric extrapolation of OS and PFS-Model Base-case with Log-logistic and Log-normal distribution

\section{Perspectives}

We compared the cost between Doc-ADT and Abi-ADT therapies from both healthcare and patient perspectives.

From the healthcare perspective, we calculated the total medical costs of Doc-ADT and Abi-ADT therapies. From the patient perspective, only patient co-payments were considered. Under the current insurance policy (coverage for urban employees and urban and rural residents), using Shanghai as a reference, an average patient co-pay rate of $25 \%$ was applied for outpatients, including all the costs associated with Abi-ADT treatment, and $15 \%$ for inpatients, including all the costs associated with Doc-ADT treatment.

\section{Sensitivity analysis}

Uncertainty is usually associated with input parameter values of an economic model, which may derive from clinical trials, observational studies or in some cases, expert opinions. Therefore, sensitivity analyses were performed to examine the stability and robustness of the results.

One-way sensitivity analysis (OWSA) was performed for all parameters. The results were recalculated by adjusting one input (parameter) at a time to determine how the model results were affected. The input was specified as multiple point estimates and was adjusted manually. Most parameters included in the one-way sensitivity
Table 2 Model inputs

\begin{tabular}{|c|c|c|}
\hline Parameters & $\begin{array}{l}\text { Base case } \\
\text { value }\end{array}$ & $\begin{array}{l}\text { Range } \\
\text { tested (\%) }\end{array}$ \\
\hline \multicolumn{3}{|l|}{ Unit drug costs (RMB) } \\
\hline Unit cost of docetaxel (cost per mg) & 73.62 & \pm 20 \\
\hline Unit cost of abiraterone (cost per mg) & 0.58 & \pm 20 \\
\hline $\begin{array}{l}\text { Unit cost of prednisolone/prednisone (cost } \\
\text { per mg) }\end{array}$ & 1.33 & \pm 20 \\
\hline \multicolumn{3}{|l|}{ Dosage } \\
\hline Daily dosage of docetaxel $\left(\mathrm{mg} / \mathrm{m}^{2}\right)$ & 75 & \pm 20 \\
\hline Daily dosage of abiraterone (mg) & 1000 & \pm 20 \\
\hline Daily dosage of prednisolone (mg) & 5 & \pm 20 \\
\hline Average height (m) & 1.615 & \pm 20 \\
\hline Average weight (kg) & 61.8 & \pm 20 \\
\hline \multicolumn{3}{|l|}{ Other medical costs (RMB) } \\
\hline Hospitalisation costs for chemotherapy & 318 & \pm 20 \\
\hline Preventive drugs for chemotherapy & 620 & \pm 20 \\
\hline ADT cost per month & 2050 & \pm 20 \\
\hline Laboratory test costs & 500 & \pm 20 \\
\hline Inpatient OOP & 15 & \pm 20 \\
\hline Outpatient OOP \% & $25 \%$ & \pm 20 \\
\hline \multicolumn{3}{|l|}{ Costs of adverse events (RMB) } \\
\hline Hypertension & 98.70 & \pm 50 \\
\hline Hypokalaemia & 65.00 & \pm 50 \\
\hline ALT increased & 225.68 & \pm 50 \\
\hline Hyperglycaemia & 115.00 & \pm 50 \\
\hline AST increased & 225.68 & \pm 50 \\
\hline Bone pain & 583.00 & \pm 50 \\
\hline Cardiac disorder & 1000.00 & \pm 50 \\
\hline Anaemia & 271.70 & \pm 50 \\
\hline Back pain & 73.60 & \pm 50 \\
\hline Spinal-cord compression & 1235.00 & \pm 50 \\
\hline Fatigue & 540.44 & \pm 50 \\
\hline Allergic reaction & 75.00 & \pm 50 \\
\hline Diarrhoea & 31.44 & \pm 50 \\
\hline Stomatitis & 74.00 & \pm 50 \\
\hline Neuropathy & 375.84 & \pm 50 \\
\hline Thromboembolism & 1794.20 & \pm 50 \\
\hline Sudden death & 3200.00 & \pm 50 \\
\hline Thrombocytopenia & 3518.04 & \pm 50 \\
\hline Neutropaenia & 720.80 & \pm 50 \\
\hline Febrile neutropaenia & 1787.20 & \pm 50 \\
\hline Infection with neutropaenia & $14,764.05$ & \pm 50 \\
\hline
\end{tabular}

analyses were adjusted by $\pm 20 \%$, and the cost data for AEs derived from clinician interviews were adjusted by $\pm 100 \%$. Because the drug cost of abiraterone used was the negotiated price, its future price cannot be increased. Therefore, only the lower limited unit cost of abiraterone acetate was considered in this analysis. The OWSA results are displayed in a tornado diagram. 
Table 3 Costs and probability of adverse events

\begin{tabular}{|c|c|c|c|c|c|}
\hline \multirow[b]{2}{*}{ Hypertension } & \multicolumn{2}{|c|}{ Abi-ADT } & \multicolumn{2}{|c|}{ Doc-ADT } & \multirow{2}{*}{$\begin{array}{l}\begin{array}{l}\text { Cost used } \\
\text { in the model }\end{array} \\
98.7\end{array}$} \\
\hline & 121 & $20.3 \%$ & & & \\
\hline Hypokalaemia & 62 & $10.4 \%$ & & & 65.0 \\
\hline Increased ALT & 33 & $5.5 \%$ & & & 225.7 \\
\hline Hyperglycaemia & 27 & $4.5 \%$ & & & 115.0 \\
\hline Increased AST & 26 & $4.4 \%$ & & & 225.7 \\
\hline Bone pain & 20 & $3.4 \%$ & & & 73.6 \\
\hline Cardiac disorder & 22 & $3.7 \%$ & & & 1000.0 \\
\hline Anaemia & 15 & $2.5 \%$ & 5 & $1.3 \%$ & 271.7 \\
\hline Back pain & 14 & $2.3 \%$ & & & 73.6 \\
\hline Spinal-cord compression & 12 & $2.0 \%$ & & & 1235.0 \\
\hline Fatigue & 10 & $1.7 \%$ & 16 & $4.1 \%$ & 540.4 \\
\hline Allergic reaction & & & 8 & $2.1 \%$ & 75.0 \\
\hline Diarrhoea & & & 4 & $1.0 \%$ & 31.4 \\
\hline Stomatitis & & & 2 & $0.5 \%$ & 74.0 \\
\hline Neuropathy & & & 4 & $1.0 \%$ & 375.8 \\
\hline Thromboembolism & & & 3 & $0.8 \%$ & 1794.2 \\
\hline Sudden death & & & 1 & $0.3 \%$ & 3200.0 \\
\hline Thrombocytopenia & & & 1 & $0.3 \%$ & 3518.0 \\
\hline Neutropaenia & & & 47 & $12.1 \%$ & 720.8 \\
\hline Febrile neutropaenia & & & 24 & $6.2 \%$ & 1787.2 \\
\hline Infection with neutropaenia & & & 9 & $2.3 \%$ & $14,764.1$ \\
\hline
\end{tabular}

To quantify the level of uncertainty in the output of the analysis, we also conducted a probabilistic sensitivity analysis (PSA). In the PSA, 1000 Monte Carlo simulations were performed to test the effect of uncertainty on the base case results for costs. Model input was specified as a distribution and was obtained from the literature. The results are displayed in a histogram for the frequency distribution of cost differences.

\section{Cost analysis results}

\section{Healthcare perspective}

The expected total costs were RMB 399,844 per patient for Doc-ADT therapy and RMB 1,286,900 per patient for Abi-ADT therapy, with a potential saving of up to RMB 887,057 per patient. Especially, the expected drug costs before clinical progression were RMB 130,786 per patient for Doc-ADT therapy, which is much lower than that for Abi-ADT therapy with RMB 1,230,951 per patient. Although the second-line treatment costs after clinical progression for Doc-ADT therapy is higher than those for Abi-ADT therapy, the total cost for Doc-ADT arm is still much lower (Table 4).

The results of the OWSA and PSA are displayed in the tornado diagram and histogram presented in Fig. 4a, b. The variables with the greatest impact on the cost difference were dosage and the unit cost of abiraterone acetate.
Table 4 Base case results from the healthcare perspective and patient perspective (unit: RMB)

\begin{tabular}{llll}
\hline & Doc-ADT & Abi-ADT & Difference \\
\hline Healthcare perspective & & & \\
Drug + ADT costs & $¥ 130,786$ & $¥ 1,230,951$ & $(¥ 1,100,165)$ \\
Medical costs & $¥ 29,098$ & $¥ 31,339$ & $(¥ 2,241)$ \\
AE costs & $¥ 266$ & $¥ 1,459$ & $(¥ 1,193)$ \\
2nd-line Tx costs & $¥ 239,694$ & $¥ 23,152$ & $¥ 216,542$ \\
Total & $¥ 399,844$ & $¥ 1,286,900$ & $(¥ 887,057)$ \\
& & & Cost saving \\
Patient perspective & & & \\
Drug + ADT costs & $¥ 27,611$ & $¥ 307,738$ & $(¥ 280,127)$ \\
Medical costs & $¥ 6,459$ & $¥ 7,835$ & $(¥ 1,376)$ \\
AE costs & $¥ 40$ & $¥ 365$ & $(¥ 325)$ \\
2nd-line Tx costs & $¥ 59,091$ & $¥ 3,473$ & $¥ 55,618$ \\
Total & $¥ 93,200$ & $¥ 319,410$ & $(¥ 226,210)$ \\
& & & Cost saving \\
\hline
\end{tabular}

In the PSA, Doc-ADT therapy was a cost-saving alternative in $100 \%$ of the simulations.

\section{Patient perspective}

The expected total out-of-pocket costs were RMB 93,200 per patient for Doc-ADT therapy and RMB 319,410 for Abi-ADT therapy, with a potential saving of up to RMB 226,210 per patient (Table 4). The results of OWSA and PSA are displayed in Fig. 4c, d).

\section{Discussion}

The network meta-analysis of Wallis et al. [19] demonstrated no significant difference in OS for patients with mHSPC treated with Doc-ADT versus Abi-ADT therapy. However, the pooled result of PFS was not reported. Our study provided an update of the Wallis study for patients with high-volume mHSPC. We used the same search strategy and extended the search period to November 2017. One additional paper reporting the PFS results from the CHAARTED trial was included. Our study found no significant difference in PFS between the two therapies.

Besides clinical effects, treatment cost is another crucial factor in choosing treatment strategies in China. Therefore, we conducted an economic evaluation to compare the costs of Doc-ADT and Abi-ADT therapies in China during the entire course of disease progression until patient death, based on their equivalent clinical effects. Our study demonstrated that Doc-ADT therapy was a cost-saving alternative to Abi-ADT therapy in Chinese patients, with potential savings of up to RMB 887,057 per patient from the healthcare perspective and RMB 226,210 per patient from the patient perspective. 


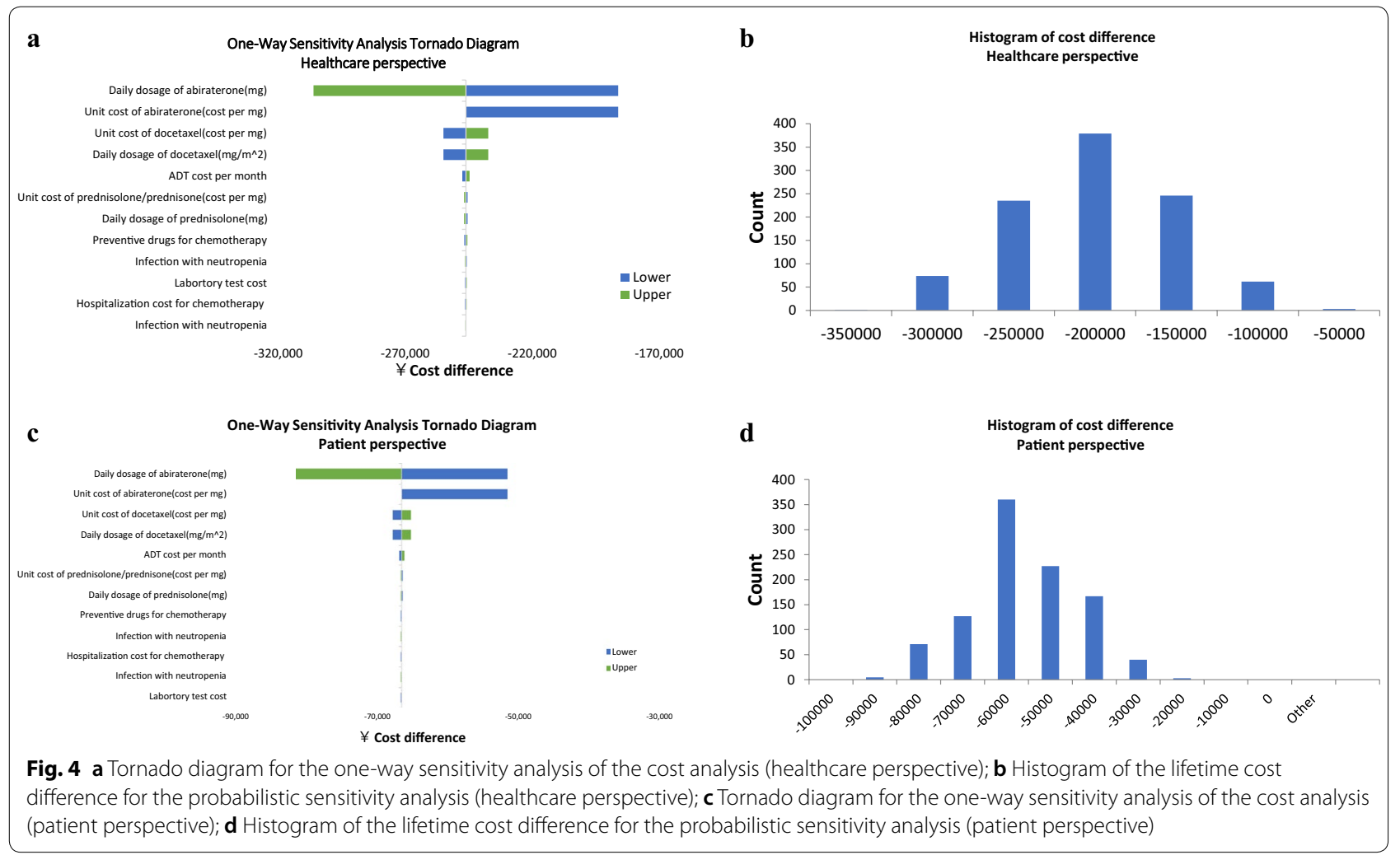

Sensitivity analyses confirmed the robustness and steadiness of these results.

Similar cost analyses of prostate cancer treatments had been assessed from the perspective of multiple countries in past few years. The STAMPEDE trial [20] in the UK compared the costs and quality of life associated with Doc-ADT and ADT therapies among men and reported that adding docetaxel to ADT was estimated to extend QALYs by 0.51 years in M1 patients (the cancer has spread to distant sites) and 0.39 years in M0 patients (no growth to distant sites). This finding demonstrated that adding the chemotherapeutic agent docetaxel to standard hormone therapy for mHSPC improves patients' overall quality of life (QoL), reduces the need for subsequent therapy, and is cost-saving. The LATITUDE study [21] compared patient-reported outcomes following Abi-ADT therapy with ADT alone in patients with newly diagnosed metastatic castration-naive prostate cancer and reported that Abi-ADT improves patients' QoL. There are also several studies assess the cost for abiraterone or docetaxel in metastatic castration-resistant prostate cancer (mCRPC) $[20,21]$, however, no study have been performed regarding with the head-to-head comparisons between Doc-ADT and Abi-ADT among patients with mHSPC.
One of the strengths of our analysis is that all cost data were collected from published literature and confirmed by local clinical experts or were directly provided by experts, reflecting current costs as well as current clinical practices in China. Furthermore, we broke down lifetime costs even beyond the clinical trial period for Doc-ADT and Abi-ADT therapies, which are presented in Table 3. For Doc-ADT therapy, we adopted the second-line treatment cost after clinical progression used in the CHAARTED trial (34\% patients continued Doc$\mathrm{ADT}$, and $66 \%$ were switched to Abi-ADT). For AbiADT therapy, since no suggestions were identified in the included trials, suggestions from local clinical experts were applied; accordingly, all patients were switched to Doc-ADT for 10 cycles. Therefore, although Doc-ADT treatment is less expensive than Abi-ADT treatment, the second-line treatment cost after clinical progression associated with the former is greater than that associated with the latter. Another strength of our analysis is that we extrapolated the PSF and OS of CHAARTED trials ADT arm survival curve to a lifetime horizon, and then applied HRs from the indirect comparison to reconstruct the curve for Abi-ADT and Doc-ADT arms. In this case, the syntheses of evidence of indirect comparison were used. 
Some limitation should be noted. First, although the method of indirect comparison was validated for comparing outcomes for RCTs, the approach is still a surrogate. Head-to-head trial comparing Doc-ADT and Abi-ADT was recommended in the future. Second, the incidence of AE for Doc-ADT and Abi-ADT were collected from the corresponding arms in the CHAARTED and LATITUDE trials, respectively. We have conducted clinician's interview, and the clinicians confirmed that there were minor differences in follow-up time and baseline characteristics between the two studies, which had little impact on the results of the cost analysis. Based on the results in the OWSA, the incidence of AEs had little effect on the results after we tested a broad range of adjustments. Third, because the patient co-pay rates for outpatients and inpatients vary across different provinces in China, we used the average patient co-pay rates in Shanghai as a reference for the patient perspective as suggested by local clinicians. Nevertheless, the sensitivity analysis verified the stability and robustness of the results.

\section{Conclusion}

Our indirect comparison shows no significant difference in either PFS or OS between these two therapies in the treatment of patients with high-volume mHSPC. The cost analysis demonstrates that Doc-ADT therapy is a cost-saving alternative to Abi-ADT in China.

\section{Summary points}

- The objective of this paper is to conduct an indirect treatment comparison of patients with high-volume mHSPC and a cost analysis between Abi-ADT and Doc-ADT therapies in China.

- The Bucher technique for indirect treatment comparison was used to compare PFS in patients treated with Abi-ADT and those treated with DocADT.

- Overall, 1895 patients from three trials reporting PFS data were included; 354 (18.7\%) patients received Doc-ADT, 597 (31.5\%) patients received Abi-ADT, and 944 (49.8\%) patients received ADT alone.

- The indirect treatment comparison demonstrated no significant difference in PFS for Abi-ADT versus Doc-ADT (HR: 0.84, 95\% CI 0.66-1.07).

- A cost analysis over a lifetime survival projection was conducted based on the updated systematic review and indirect comparison. The related medical cost was derived from the local setting.

- The cost analysis indicated that Doc-ADT therapy costs less than Abi-ADT, with potential savings of up to RMB 887,057 per patient from the healthcare perspective and RMB 226,210 per patient from the patient perspective.

- No significant differences in either PFS or OS exist between Doc-ADT and Abi-ADT therapy for patients with high-volume mHSPC. The cost analysis revealed that Doc-ADT therapy is a cost-saving alternative to Abi-ADT in China.

\section{Acknowledgements}

High tribute shall be paid to Dr. Xiang Li from Huaxi Hospital, Dr. Yao Zhu from Fudan University Shanghai Cancer Center, Dr. Xinrong Fan from Beijing Union Hospital, Dr. Yonghong Li from Sun-Yat-Sen University Cancer Center, and Dr. Zhiquan Hu from Wuhan Tongji Hospital for their expert opinions on relevant local cost data inputs in the analysis. We also sincerely thank Dr. Tengbin Xiong for his contribution in methodology advice in this project, and Dr. Li Liu and Dr. Yang Xie for their contribution to help review the manuscript.

\section{Authors' contributions}

All of the authors contributed significantly to the collection and analysis of the data, reviewed and edited the manuscript, and agree with its content and submission for publication. The authors are fully responsible for all content and editorial decisions. All authors read and approved the final manuscript.

\section{Funding}

This study was funded by Sanofi China.

\section{Availability of data and materials}

Data sharing is not applicable to this article as no datasets were generated or analysed during the current study.

\section{Ethics approval and consent to participate}

Not applicable.

\section{Consent for publication}

Not applicable.

\section{Competing interests}

Chaoyun Li and Xingxing Yao are employees of Sanofi China. Shuli Qu, Yanjun Liu are employees of IQVIA, which received research funding from Sanofi China. The authors declare that they have no competing interests.

\section{Author details}

${ }^{1}$ Department of Pharmacy, Beijing Hospital, Beijing, China. ${ }^{2}$ Real World Solutions, IQVIA, Shanghai, China. ${ }^{3}$ Health Economics \& Outcome Research, Sanofi, Shanghai, China. ${ }^{4}$ Department of Urology, Beijing Hospital, No. 1 Dongdan Dahua Road, Dongcheng District, Beijing 100730, China.

Received: 4 November 2018 Accepted: 15 November 2019

Published online: 13 December 2019

\section{References}

1. Fitzmaurice C, Allen C, Barber RM, Barregard L, Bhutta ZA, Brenner H, et al. Global, regional, and national cancer incidence, mortality, years of life lost, years lived with disability, and disability-adjusted life-years for 32 cancer groups, 1990 to 2015: a systematic analysis for the global burden of disease study. JAMA Oncol. 2017;3(4):524-48.

2. Torre LA, Bray F, Siegel RL, Ferlay J, Lortet-Tieulent J, Jemal A. Global cancer statistics, 2012. CA. 2015;65(2):87-108.

3. Chen W, Zheng R, Baade PD, Zhang S, Zeng H, Bray F, et al. Cancer statistics in China, 2015. CA. 2016;66(2):115-32.

4. Han S, Zhang S, Chen W, Li C. Analysis of the status and trends of prostate cancer incidence in China. Chin Clin Oncol. 2013;18(4):330-4.

5. Sweeney CJ, Chen YH, Carducci M, Liu G, Jarrard DF, Eisenberger M, et al. Chemohormonal therapy in metastatic hormone-sensitive prostate cancer. N Engl J Med. 2015;373(8):737-46. 
6. Qin XJ, Ma CG, Ye DW, Yao XD, Zhang SL, Dai B, et al. Tumor cytoreduction results in better response to androgen ablation-a preliminary report of palliative transurethral resection of the prostate in metastatic hormone sensitive prostate cancer. Urol Oncol. 2012;30(2):145-9.

7. Mottet N, De Santis M, Briers E, Gillessen S, Grummet JP, Lam TB, et al. Updated guidelines for metastatic hormone-sensitive prostate cancer: abiraterone acetate combined with castration is another standard. Elsevier; 2017.

8. Fizazi K, Tran N, Fein L, Matsubara N, Rodriguez-Antolin A, Alekseev BY, et al. Abiraterone plus prednisone in metastatic, castration-sensitive prostate cancer. N Engl J Med. 2017;377(4):352-60

9. Gravis G, Boher J-M, Joly F, Soulié M, Albiges L, Priou F, et al. Androgen deprivation therapy (ADT) plus docetaxel versus ADT alone in metastatic non castrate prostate cancer: impact of metastatic burden and long-term survival analysis of the randomized phase 3 GETUG-AFU15 trial. Eur Urol. 2016;70(2):256-62.

10. Booth CM, Eisenhauer EA. Progression-free survival: meaningful or simply measurable? J Clin Oncol. 2012;30(10):1030-3.

11. Jakovljevic MM. Comparison of historical medical spending patterns among the BRICS and G7. J Med Econ. 2016;19(1):70-6.

12. Jakovljevic M, Yamada T. Role of health economic data in policy making and reimbursement of new medical technologies. Front Pharmacol. 2017;8:662.

13. Higgins JPTAD, Gøtzsche PC, et al. The Cochrane Collaboration's tool for assessing risk of bias in randomised trials. BMJ. 2011;343:d5928.

14. Guyot P, Ades AE, Ouwens MJ, Welton NJ. Enhanced secondary analysis of survival data: reconstructing the data from published Kaplan-Meier survival curves. BMC Med Res Methodol. 2012;12:9.

15. Sweeney C, Chen Y, Liu G, Carducci M, Jarrard D, Eisenberger M, et al. Long term efficacy and QOL data of chemohormonal therapy (C-HT) in low and high volume hormone naïve metastatic prostate cancer (PrCa): E3805 CHAARTED trial. Ann Oncol. 2016;27(6):243-65. https://doi. org/10.1093/annonc/mdw372.
16. Bucher HC, Guyatt GH, Griffith LE, Walter SD. The results of direct and indirect treatment comparisons in meta-analysis of randomized controlled trials. J Clin Epidemiol. 1997;50(6):683-91.

17. Wells G, Sultan S, Chen L, Khan M, Coyle D. Indirect evidence: indirect treatment comparisons in meta-analysis. Ottawa: Can Agency Drugs Technol Health; 2009. p. 1-94.

18. Lin X. The report on nutrition and chronic diseases of Chinese residents. J Chin Med Manag. 2015;23(13):89.

19. Wallis CJD, Klaassen Z, Bhindi B, Goldberg H, Chandrasekar T, Farrell $\mathrm{AM}$, et al. Comparison of abirdsaterone acetate and docetaxel with androgen deprivation therapy in high-risk and metastatic hormone-naive prostate cancer: a systematic review and network meta-analysis. Eur Urol. 2017;73(6):834-44.

20. Nicholas James BW, Eleftherios Sideris, Melissa Ruth Spears, David P. Dearnaley, Malcolm Mason, Noel Clarke, Mahesh K B Parmar, Matthew Robert Sydes, Mark Sculpher. Addition of docetaxel to first-line long-term hormone therapy in prostate cancer (STAMPEDE): Long-term survival, quality-adjusted survival, and cost-effectiveness analysis. American Society of Clinical Oncology 2018 Annual meeting. 2018.

21. Chi KN, Protheroe A, Rodríguez-Antolín A, Facchini G, Suttman H, Matsubara $\mathrm{N}$, et al. Patient-reported outcomes following abiraterone acetate plus prednisone added to androgen deprivation therapy in patients with newly diagnosed metastatic castration-naive prostate cancer (LATITUDE): an international, randomised phase 3 trial. Lancet Oncol. 2018;19(2):194-206.

\section{Publisher's Note}

Springer Nature remains neutral with regard to jurisdictional claims in published maps and institutional affiliations.
Ready to submit your research? Choose BMC and benefit from:

- fast, convenient online submission

- thorough peer review by experienced researchers in your field

- rapid publication on acceptance

- support for research data, including large and complex data types

- gold Open Access which fosters wider collaboration and increased citations

- maximum visibility for your research: over $100 \mathrm{M}$ website views per year

At BMC, research is always in progress.

Learn more biomedcentral.com/submissions 